\title{
Domaines d'existence et de cristallisation de borates et aluminoborates de gadolinium
}

\author{
O. Aloui-Lebbou, C. Goutaudier et M.T. Cohen-Adad \\ Laboratoire de Physico-Chimie des Matériaux Luminescents, UMR CNRS 5620, \\ Université Claude Bernard Lyon I, 69622 Villeurbanne Cedex, France
}

\begin{abstract}
Rare earth borates and aluminoborates single crystals were investigated for their applications as laser host materials and scintillators. The investigation of the ternary system $\mathrm{Ln}_{2} \mathrm{O}_{3}-\mathrm{Al}_{2} \mathrm{O}_{3}-\mathrm{B}_{2} \mathrm{O}_{3}(\mathrm{Ln}=\mathrm{Gd})$ was undertaken in order to determine the thermal behavior, the best conditions of the growth from a melt and their ability to be reproduced. In first, the different solid phases formed in the binary system $\mathrm{Gd}_{2} \mathrm{O}_{3}-\mathrm{B}_{2} \mathrm{O}_{3}$ were studied and then the crystallization field of each phase was defined as a function of composition and temperature.
\end{abstract}

\section{INTRODUCTION}

Les matrices à base d'ions borates $\mathrm{BO}_{3}{ }^{3-}$ sont connues pour leurs applications sous forme de poudre dans le domaine des lampes et des écrans de visualisation [1] ; elles sont également susceptibles d'applications comme matériaux lasers ou matériaux scintillateurs pour la détection de rayonnements ionisants de haute énergie (rayons $\gamma$ )[2]. Ces applications nécessitent la mise au point de procédés d'élaboration de monocristaux optimisés en taille et qualité. Cependant, l'obtention de monocristaux se heurte à d'importantes difficultés liées à la température de fusion élevée des composés intervenant dans les systèmes $\mathrm{R}_{2} \mathrm{O}_{3}-\mathrm{B}_{2} \mathrm{O}_{3}$ et $\mathrm{R}_{2} \mathrm{O}_{3}-\mathrm{Al}_{2} \mathrm{O}_{3}-\mathrm{B}_{2} \mathrm{O}_{3}$ où $\mathrm{R}$ est une terre rare, à la volatilité de l'oxyde de bore à haute température et à l'existence de transitions de phases. La connaissance des domaines de cristallisation de ces composés et de leur comportement thermique est donc un préalable indispensable pour définir des conditions de croissance à plus basse température. Le travail a été focalisé sur les borates à base de gadolinium. Une première étude du système binaire $\mathrm{Gd}_{2} \mathrm{O}_{3}-\mathrm{B}_{2} \mathrm{O}_{3}$ [3] avait confirmé l'existence des trois composés : $\mathrm{GdBO}_{3}, \mathrm{Gd}_{3} \mathrm{BO}_{6}$ et $\mathrm{Gd}\left(\mathrm{BO}_{2}\right)_{3}$, et donné une première approche de leur domaine d'existence. Parce qu'il restait des ambiguïtés, ce travail reprend l'étude des domaines de stabilité des borates simples appartenant au système binaire puis dans un second temps, l'étude de l'aluminoborate $\mathrm{GdAl}_{3}\left(\mathrm{BO}_{3}\right)_{4}$ et le développement de son domaine de cristallisation dans le ternaire $\mathrm{Gd}_{2} \mathrm{O}_{3}-\mathrm{Al}_{2} \mathrm{O}_{3}-\mathrm{B}_{2} \mathrm{O}_{3}$ en relation avec les domaines d'existence des phases adjacentes.

\section{CONDITIONS EXPERIMENTALES}

Tous les échantillons étudiés ont été préparés par réaction à l'état solide à partir d'oxyde de gadolinium, d'alumine dans le cas des borates mixtes et d'acide borique en léger excès par rapport à la stoechiométrie afin de compenser la vaporisation de $\mathrm{B}_{2} \mathrm{O}_{3}$ à haute température. Après broyage et compactage des poudres, les échantillons ont été traités thermiquement sous atmosphère d'oxygène $\left(\mathrm{PO}_{2}=1\right.$ bar $)$. Plusieurs cycles ont été nécessaires pour obtenir les composés à l'état monophasé, la composition nominale des échantillons étant maintenue constante par addition de $\mathrm{B}_{2} \mathrm{O}_{3}$ après chaque traitement thermique. La quantité d'acide borique à ajouter était calculée à partir de la masse des échantillons avant et après le traitement thermique. En fin d'élaboration, la composition réelle des échantillons était contrôlée à partir de la mesure des pertes globales en $\mathrm{B}_{2} \mathrm{O}_{3}$. L'identification des phases et leur comportement en fonction de la température ont été suivis par diffraction des rayons $\mathrm{X}$ et analyse thermogravimétrique sous flux $\mathrm{d}^{\prime} \mathrm{O}_{2}\left(\mathrm{P}=1 \mathrm{bar}\right.$, vitesse de chauffe et refroidissement : $\left.5^{\circ} \mathrm{C} \cdot \mathrm{mn}^{-1}\right)$. Les résultats d'analyse thermique sont donnés en composition réelle. 


\section{RESULTATS ET DISCUSSION}

\subsection{Système binaire $\mathrm{Gd}_{2} \mathrm{O}_{3}-\mathrm{B}_{2} \mathrm{O}_{3}$}

La figure 1 présente l'ensemble des équilibres observés dans ce système entre la température ambiante et $1650^{\circ} \mathrm{C}$, dans le domaine de composition où se manifestent les composés de stoechiométrie $\operatorname{Gd}\left(\mathrm{BO}_{2}\right)_{3}$, $\mathrm{GdBO}_{3}$ et $\mathrm{Gd}_{3} \mathrm{BO}_{6}$.

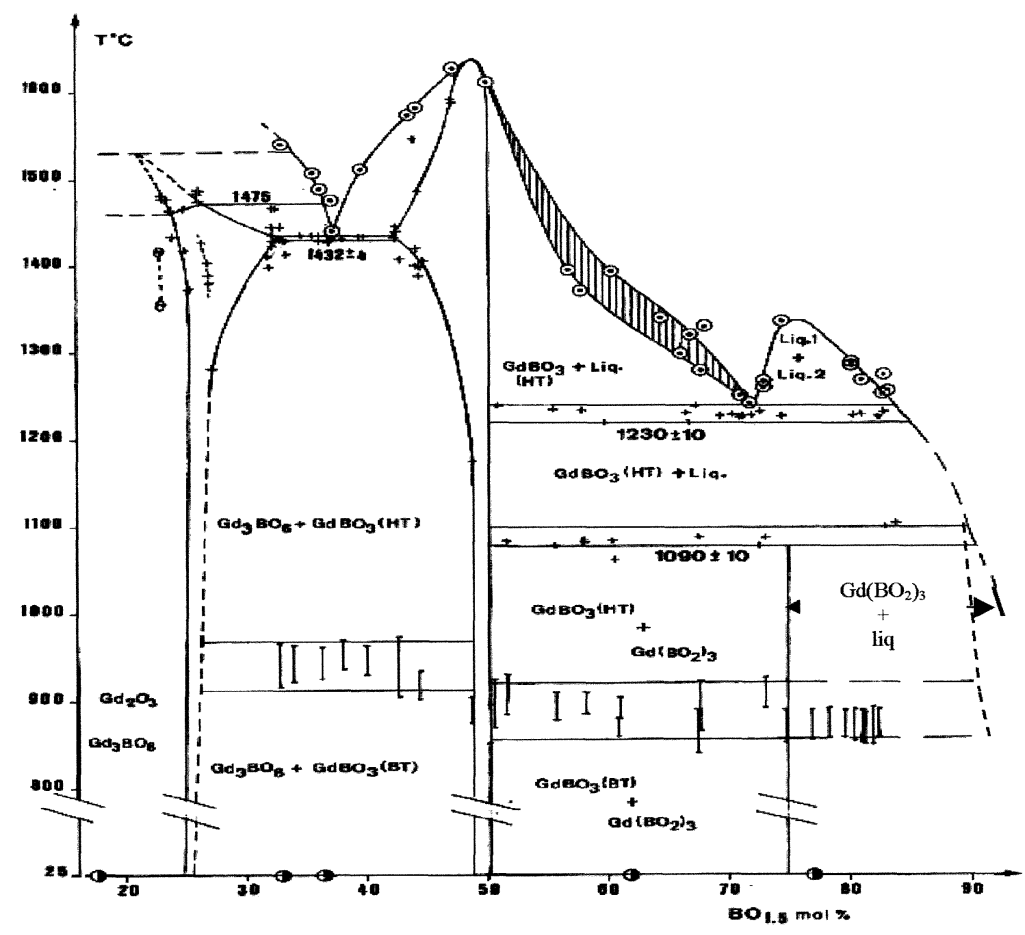

Figure 1 : Système binaire $\mathrm{Gd}_{2} \mathrm{O}_{3}-\mathrm{B}_{2} \mathrm{O}_{3}$ sous la pression atmosphérique

L'orthoborate $\mathrm{GdBO}_{3}$ fond de façon congruente au voisinage de $1635 \pm 7^{\circ} \mathrm{C}$, la composition à la congruence (environ $49.0 \mathrm{~mol} \%$ en $\mathrm{BO}_{1.5}$ ) étant légèrement décalée par rapport à la stoechiométrie. Entre 875 et $940^{\circ} \mathrm{C}$, il présente une transition structurale renversable avec forte hystérèse au refroidissement. La différence de température de transition observée de part et d'autre du rapport $\mathrm{Gd} / \mathrm{B}=1$ traduit l'existence d'un étroit domaine d'homogénéité vers $1000^{\circ} \mathrm{C}$ qui s'élargit jusqu'à $8 \mathrm{~mol} \%$ en $\mathrm{BO}_{1.5}$ à $1432^{\circ} \mathrm{C}$. $\mathrm{GdBO}_{3}$ intervient dans deux réactions d'équilibre de type eutectique :

- un équilibre monotectique vers $1230 \pm 10^{\circ} \mathrm{C}$ correspondant à la réaction Liq $1 \Leftrightarrow \mathrm{GdBO}_{3}+\mathrm{Liq} 2$, la compositions des liquides étant respectivement 72,0 et $84,0 \mathrm{~mol} \% \mathrm{BO}_{1.5}$,

- un eutectique vrai vers $1432 \pm 4^{\circ} \mathrm{C}$ selon la réaction $\mathrm{Liq} \Leftrightarrow \mathrm{GdBO}_{3}+\mathrm{Gd}_{3} \mathrm{BO}_{6}$.

Le composé stoechiométrique $\mathrm{Gd}\left(\mathrm{BO}_{2}\right)_{3}$ se décompose de façon péritectique à $1090^{\circ} \mathrm{C}$ en donnant un liquide très riche en oxyde de bore et le solide $\mathrm{GdBO}_{3}$.

Le composé $\mathrm{Gd}_{3} \mathrm{BO}_{6}$ présente également un domaine d'homogénéité à basse température. Ce domaine s'élargit de part et d'autre à partir de $1200^{\circ} \mathrm{C}$ et du côté riche en $\mathrm{B}_{2} \mathrm{O}_{3}$, il s'étend jusqu'à la composition de $32.8 \mathrm{~mol} \%$ en $\mathrm{BO}_{1.5}$. Au voisinage de $1475^{\circ} \mathrm{C}$, il existe une transition structurale confirmé par un changement drastique des diffractogrammes RX entre 1450 et $1500^{\circ} \mathrm{C}$. D'autre part, la forme des courbes de solidus laisse penser que $\mathrm{Gd}_{3} \mathrm{BO}_{6}$ se décomposerait de façon péritectique vers $1530^{\circ} \mathrm{C}$. 


\subsection{Système ternaire $\mathrm{Gd}_{2} \mathrm{O}_{3}-\mathrm{Al}_{2} \mathrm{O}_{3}-\mathrm{B}_{2} \mathrm{O}_{3}$}

Le système a été approximativement défini à partir de deux coupes isopléthiques passant par la stoechiométrie $\mathrm{GdAl}_{3}\left(\mathrm{BO}_{3}\right)_{4}$ et correspondant aux rapports $\mathrm{Gd} / \mathrm{Al}=0.25$ (coupe $\mathrm{I}$ ) et $\mathrm{Al} / \mathrm{B}=1$ (coupe II). Ces deux coupes montrent que contrairement aux données de la littérature[4], le composé $\mathrm{GdAl}_{3}\left(\mathrm{BO}_{3}\right)_{4}$ présente sans ambiguïté une fusion congruente à $1265^{\circ} \mathrm{C}$. Il en résulte que la partie de la coupe I représentative des compositions supérieures à $50 \mathrm{~mol} \%$ en $\mathrm{BO}_{1.5}$ est quasi binaire et que deux domaines d'équilibre liq $+\mathrm{GdAl}_{3}\left(\mathrm{BO}_{3}\right)_{4}$ apparaissent entre la fusion et $384^{\circ} \mathrm{C}$, séparés par un palier quasimonotectique à $1219 \pm 10^{\circ} \mathrm{C}$ traduisant l'équilibre Liq.1 $\Leftrightarrow$ Liq.2 $+\mathrm{GdAl}_{3}\left(\mathrm{BO}_{3}\right)_{4}$.

Au-dessous de $384^{\circ} \mathrm{C}$, se développe un domaine d'équilibre solide-solide faisant intervenir $\mathrm{GdAl}_{3}\left(\mathrm{BO}_{3}\right)_{4}$ et $\mathrm{B}_{2} \mathrm{O}_{3}$ conduisant à attribuer les accidents thermiques observés à $384^{\circ} \mathrm{C}$ à un équilibre eutectique liquide $\Leftrightarrow \mathrm{GdAl}_{3}\left(\mathrm{BO}_{3}\right)_{4}+\mathrm{B}_{2} \mathrm{O}_{3}$.

De la séquence des phases observées dans la portion d'isoplèthe comprise entre 50 et $20 \mathrm{~mol} \%$ en $\mathrm{BO}_{1.5}$, il résulte que :

- trois domaines de cristallisation se manifestent, ils sont relatifs à $\mathrm{GdAl}_{3}\left(\mathrm{BO}_{3}\right)_{4}, \mathrm{GdBO}_{3}$ et $\mathrm{Al}_{18} \mathrm{~B}_{4} \mathrm{O}_{33}$,

- un palier à $1223 \pm 7^{\circ} \mathrm{C}$ doit être attribué à l'équilibre transitoire Liquide $+\mathrm{GdBO}_{3} \Leftrightarrow \mathrm{GdAl}_{3}\left(\mathrm{BO}_{3}\right)_{4}+$ $\mathrm{Al}_{18} \mathrm{~B}_{4} \mathrm{O}_{33}$.

Au-dessous de $1223^{\circ} \mathrm{C}$, la phase liquide disparaît et les trois phases solides seront observées jusqu'à 29,6 mol\% en $\mathrm{BO}_{1.5}$ où la trace de la coupe isopléthique dans le plan des compositions croise la droite joignant les points représentatifs des phases $\mathrm{GdBO}_{3}$ et $\mathrm{Al}_{18} \mathrm{~B}_{4} \mathrm{O}_{33}$.

En deçà de $29,6 \mathrm{~mol} \%$ en $\mathrm{BO}_{1.5}, \mathrm{GdAl}_{3}\left(\mathrm{BO}_{3}\right)_{4}$ disparaît et les seules phases solides identifiées ont été $\mathrm{GdBO}_{3}$ et $\mathrm{Al}_{18} \mathrm{~B}_{4} \mathrm{O}_{33}$.

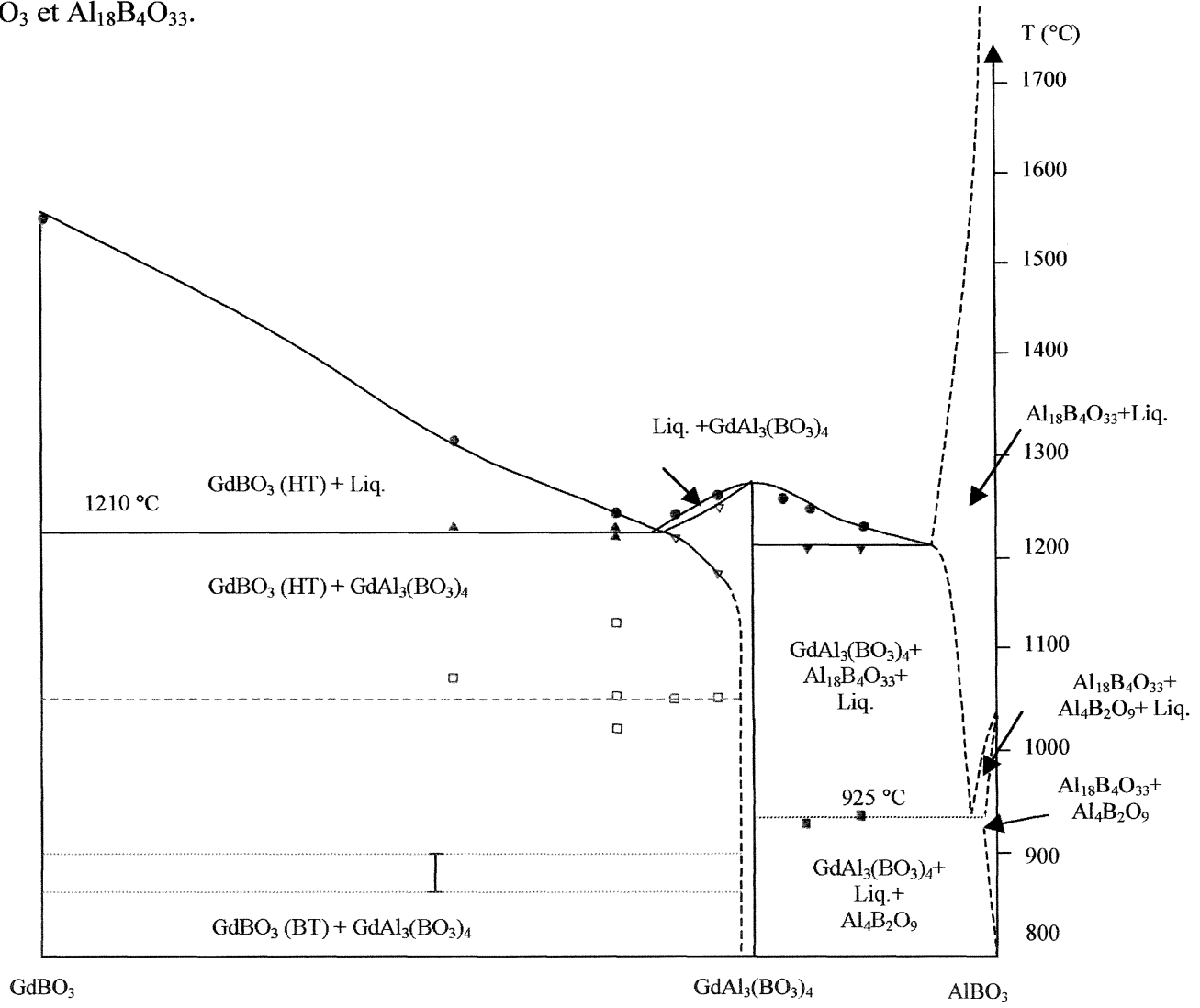

Figure 2 : Coupe II correspondant à $50 \mathrm{~mol} \% \mathrm{BO}_{1.5}$ dans le système ternaire $\mathrm{Gd}_{2} \mathrm{O}_{3}-\mathrm{Al}_{2} \mathrm{O}_{3}-\mathrm{B}_{2} \mathrm{O}_{3}$ 
Le long de la coupe II se développe également un domaine de non stoechiométrie pour $\mathrm{GdAl}_{3}\left(\mathrm{BO}_{3}\right)_{4}$ étendu vers les compositions riches en Gd.

Aucune modification structurale de la phase $\mathrm{GdAl}_{3}\left(\mathrm{BO}_{3}\right)_{4}$ n'a été observée entre la température ambiante et la fusion et tous les domaines d'équilibre ont été identifiés.

\subsection{Domaines de cristallisation}

La figure 3 rassemble ces résultats et décrit, en projection sur le plan des compositions, le domaine de cristallisation des phases solides $\mathrm{GdBO}_{3}$ et $\mathrm{GdAl}_{3}\left(\mathrm{BO}_{3}\right)_{4}$. Ces domaines sont adjacents à ceux des phases $\mathrm{Al}_{18} \mathrm{~B}_{4} \mathrm{O}_{33}, \mathrm{~B}_{2} \mathrm{O}_{3}, \mathrm{Gd}\left(\mathrm{BO}_{2}\right)_{3}$ et $\mathrm{Al}_{4} \mathrm{~B}_{2} \mathrm{O}_{9}$ ainsi qu'au domaine de démixtion liquide-liquide.

Cette projection montre que ces domaines de cristallisation ne sont pas favorables à la croissance de monocristaux par simple refroidissement d'un mélange fondu :

pour $\mathrm{GdBO}_{3}$, les températures de cristallisation étant trop élevées, le mélange se détitrera en $\mathrm{B}_{2} \mathrm{O}_{3}$, pour $\mathrm{GdAl}_{3}\left(\mathrm{BO}_{3}\right)_{4}$, malgré un développement du domaine de cristallisation jusqu'à $384^{\circ} \mathrm{C}$ (du côté riche en $\mathrm{B}_{2} \mathrm{O}_{3}$ ), la composition du liquidus est trop appauvrie pour que le refroidissement produise des cristaux de dimension acceptable, en accord avec les résultats reportés dans la littérature[5].

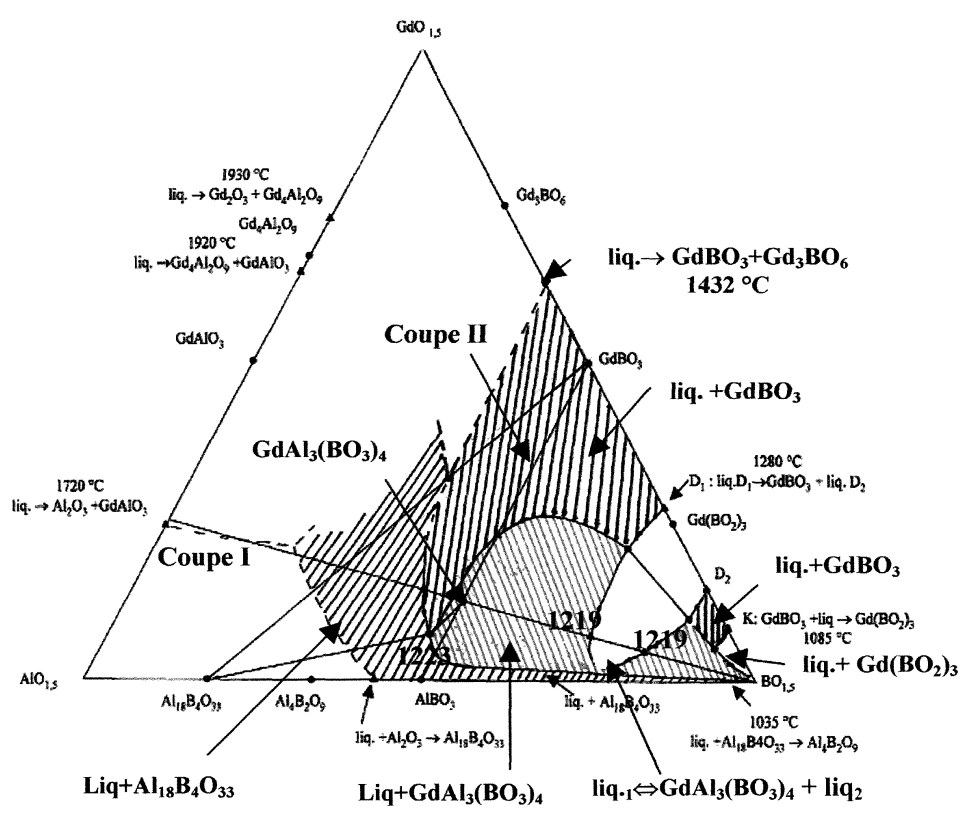

Figure 3 : Domaines de cristallisation des différentes phases solides

\section{Références}

1. M. Leskela, L. Niinisto, Hanbook of the physics and chemistry of rare earth, Eds K.A. Gschneidner and L. Eyring (Elsevier Science publishers B.V., 1986), p.203

2. L. Zhang et al., Proc. of Int. Conf. on Inorg. Scintillators, Shangaï, PR China, sept.22-25 (1997) 303

3. M.T. Cohen-Adad, O. Aloui, C. Goutaudier, G. Panczer, C. Dujardin, C. Pedrini, P. Florian, D. Massiot, F. Gerard, C. Kappenstein, J. Solid State Chem., 154, 204-213 (2000)

4. E.Koporulina, N.Y. Leonyuk, S.N.Barilo, L.A. Kurnevich, J. Cryst. Growth, 198/199, 460 (1999)

5. N.I. Leonyuk, E.V. Koporulina, S.N. Barilo, L.A. Kurnevich, G.L. Bychkov, J. Cryst. Growth, 191, $135(1998)$ 